\title{
Relation Between Grain Shape and Fractal Properties in Random Apollonian Packing with Grain Rotation
}

\author{
Gary W. Delaney, ${ }^{1,2}$ Stefan Hutzler, ${ }^{2}$ and Tomaso Aste $^{1}$ \\ ${ }^{1}$ Department of Applied Mathematics, Australian National University, Canberra, Australia \\ ${ }^{2}$ School of Physics, Trinity College Dublin, Ireland
}

(Received 9 December 2007; revised manuscript received 11 April 2008; published 16 September 2008)

\begin{abstract}
We extend the common theme of random Apollonian packing of circles to consider orientable grains with a noncircular shape. Systems of up to $10^{6}$ grains are examined for a range of polygonal and elliptical shapes using both the random Apollonian packing model and the new rotational random Apollonian packing model which takes into account the extra rotational degree of freedom of noncircular grains. We identify the constraining length $D_{c}$ that limits growth of the grain during the packing process and find that a universal relation exists between grain shape and the scaling properties of the system.
\end{abstract}

Scientists have long considered space filling problems in which nonoverlapping units of smaller and smaller sizes are placed according to a given set of rules [1-4]. The oldest known packing of this kind is the Apollonian packing (AP) of circles introduced by Apollonius of Perga around 200 B.C. [5]. This packing is formed by placing a circular disk in the space between three mutually touching disks so that it just touches the other three. The procedure is then continually repeated, filling the new gaps generated by the addition of each new disk. Apollonian packing leads to a dense system, with the size of the circles becoming smaller and smaller and the packing fraction approaching $\phi=1$ in the limit of an infinite number of disks. The AP model was generalized by Manna [6,7] to consider the case where the centers of the disks are chosen randomly (RAP, Fig. 1). This model was further extended by Andrienko, Brilliantov, and Krapivsky (ABK model) to allow multiple disks to nucleate simultaneously with a linear growth rate [8-10]. Dodds and Weitz [11] showed some of the universal features of these models (AP, RAP, ABK, etc.), considering them in terms of a broad class which they refer to as "packing-limited growth" (PLG) models. In PLG models, grains are seeded randomly, they grow according to a given rule, and they stop growing when they collide with another grain. Dodds and Weitz highlighted the fact that the RAP and $\mathrm{ABK}$ models converge as the number of placed grains increases. This is the consequence of a coupled mechanism where the number of pores increases and their sizes decrease. Thus for the ABK model, collisions of growing grains increasingly occur with already placed grains (the sole mechanism of packing in the RAP model), while collisions with other growing grains become increasingly unlikely.

In PLG models, the inverse cumulate distribution $N(r)$ of the grain sizes (number of grains with sizes larger than or equal to $r$ ) in the limit of small sizes behaves accordingly with

$$
N(r)=n_{0}\left(\frac{r}{r_{0}}\right)^{1-\alpha}
$$

where $n_{0}$ is the number of grains at which the relation becomes valid and $r_{0}$ is the effective grain size at which this takes place. The decay of the pore space volume ( $\rho=$ $1-\phi$ ) with the total number of packed elements $n$ is also described by a power law (for large $n$ ) with

$$
\rho(n)=\rho_{0}\left(\frac{n}{n_{0}}\right)^{-\beta},
$$

where $\rho_{0}$ is the pore space volume at which the relation

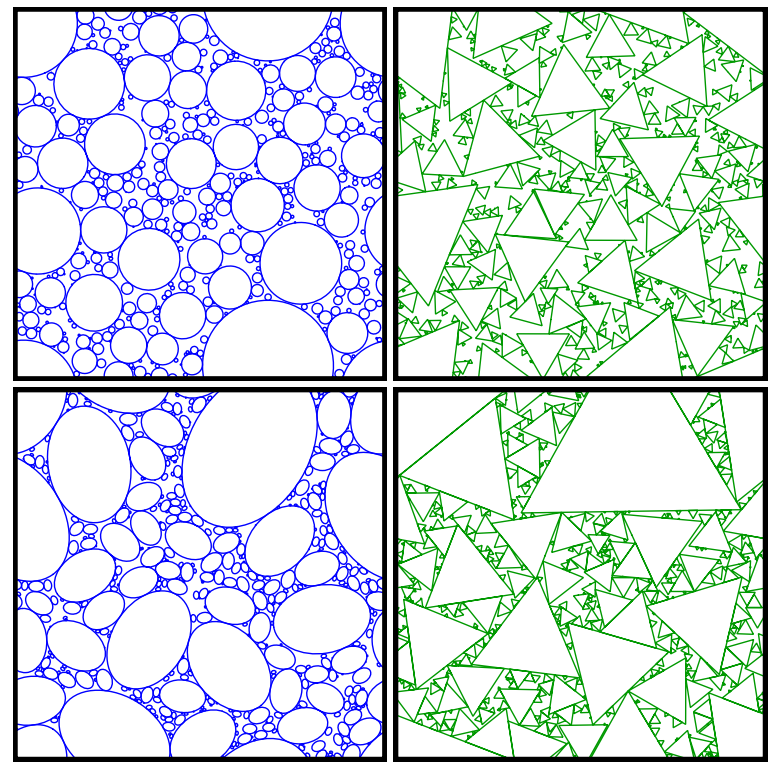

FIG. 1 (color online). RAP packings of 500 circles (top left) and triangles (top right). RRAP packing of 500 ellipses with aspect ratio $\lambda=0.7$ (bottom left) and RRAP packing of triangles (bottom right). Grains pack more densely in the RRAP model as a result of being able to rotate during the packing process. 
becomes valid when $n_{0}$ grains have been packed. It has been shown [11] that the two exponents $\alpha$ and $\beta$ are related by $\alpha=1+\frac{2}{\beta+1}$, and we have verified that this relation also holds for polygonal and elliptical shapes in both the RAP and rotational random Apollonian packing (RRAP) models (Fig. 2). We refer to $\beta$ as the packing efficiency exponent as it is a measure of how quickly a particular shape fills space. Combining this expression for $\alpha$ with Eqs. (1) and (2), one can verify that the fractal dimension is $D_{f}=\frac{2}{\beta+1}$.

Dodds and Weitz obtained numerical estimates of the scaling exponents $\alpha$ and $\beta$ for the RAP model, and the ABK model for the cases of disks growing heterogeneously, exponentially and linearly. They find a universal exponent $\alpha \simeq 2.56$, independent of the growth dynamics. In a further study Dodds and Weitz consider the role of shape in a RAP model consisting of grains composed of squares, rectangles, and grains formed from combining sets of rectangles [12]. They find that the exponent $\alpha$ is shape dependent, taking values in the range of $2.56 \leq$ $\alpha \leq 3$.

In this work we consider a model in which grains can have a noncircular shape and consider packings with a range of polygonal and elliptical shapes. We examine the detailed role that shape plays in determining the properties of the generated packings. We first achieve this by generating RAP packings of grains [12], for a range of different shapes, with each grain seeded with a randomly determined angle of orientation. This approach, however, ignores the additional degree of freedom of noncircular grains. In the RAP model, a grain stops growing on collision with another grain. However, a larger nonoverlapping grain can be placed, if the grain is allowed to take advan-

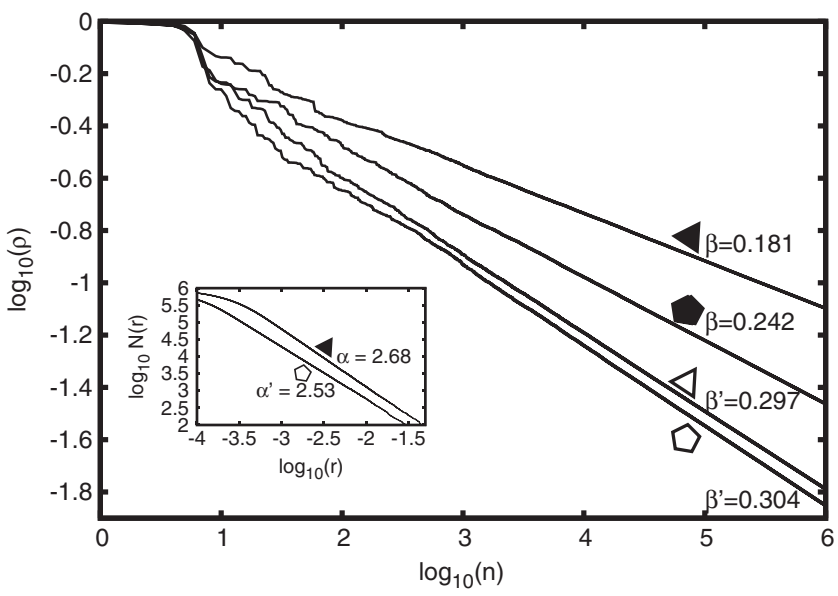

FIG. 2. The decay of the pore space volume for triangles and pentagons for $n=10^{6}$ in both the RAP (filled symbols) and RRAP (open symbols) models. Inset shows inverse cumulate distribution $N(r)$ of the grain sizes. The clear power-law regions verify the validity of Eqs. (1) and (2) in both RAP and RRAP models. tage of its additional rotational degree of freedom, choosing the orientation about its center point which allows the largest grain size to be placed (Fig. 1). We term this new model rotational random Apollonian packing. Simulations are initially seeded with 4 grains at random positions and orientations and all simulations use periodic boundary conditions. For both models we find that Eqs. (1) and (2) are verified (Fig. 2). For each shape, we determine the exponents $\beta$ (RAP) and $\beta^{\prime}$ (RRAP) by fitting the powerlaw regions of $\log \rho(n)$ vs $\log n$. A clear power-law behavior is evident when $n>1000$ grains have been packed, as shown by the linear trends at large $n$. Multiple packings of $n=10^{6}$ were generated for shapes in both the RAP and RRAP models. The data were averaged and the standard deviation of the estimate of $\beta$ and $\beta^{\prime}$ for each grain shape was determined. Smaller packings of $n=10^{5}$ were also generated and it was verified that the packing efficiency exponents coincide with those for $n=10^{6}$.

As an initial verification of our simulation we determined $\beta$ from RAP simulations of circles and of squares, finding $\beta=0.277(2)$ for circles and $\beta=0.224(2)$ for squares. (The numbers in parentheses indicate the standard deviation of our estimates for the last digit.) These are consistent with the values of $\beta=0.278(1)$ (circles) and $\beta=0.223$ (2) (squares) found by Dodds and Weitz [11,12].

Then we consider the logical progression of $s$-sided polygons that are circumscribed by a circle: $s=3$ (equilateral triangle), $s=4$ (square), $s=5$ (pentagon), and so on, with $s \rightarrow \infty$ tending toward a circle. The variation of the exponents $\beta$ and $\beta^{\prime}$ for this set of shapes is shown in Fig. 3. They show a very interesting behavior as the number of edges is increased. For the RAP case, the exponent $\beta$ has a quite smooth increase from $\beta=0.181(2)$ for $s=3$ to a value of $\beta=0.275(2)$ for $s=20$. A very different behavior is found for our new RRAP model. The maximum $\beta^{\prime}=$ $0.328(2)$ is found for square grains and this is followed by a slow decrease with $\beta^{\prime}=0.279$ (2) found for $s=20$. The

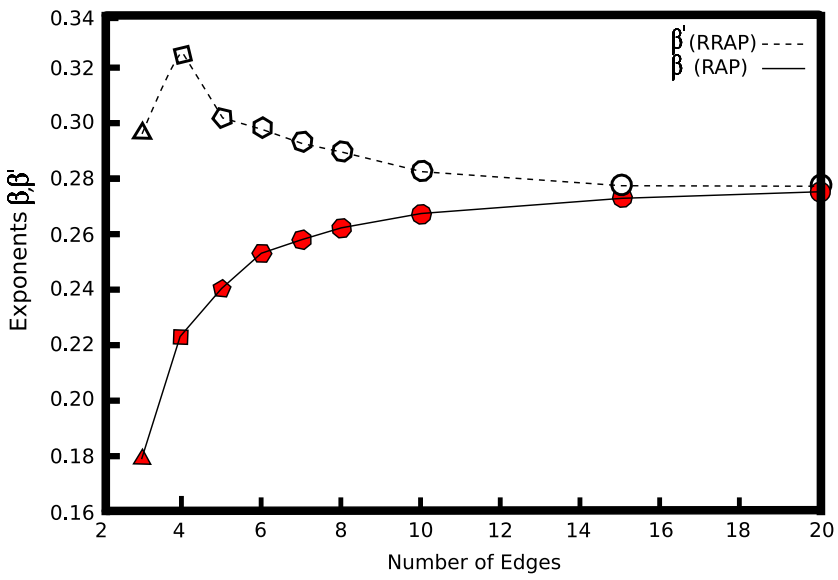

FIG. 3 (color online). Variation of the packing efficiency exponents $\beta$ (filled symbols) and $\beta^{\prime}$ (open symbols) as the number of edges of the packed grains increases. 
data clearly show that both the RAP and RRAP models converge towards the value for circles $[\beta=0.277(2)]$, as expected when the number of edges is increased and the additional rotational degree of freedom becomes unimportant.

We have also considered elliptical grains, again generating packings using both the RAP and RRAP models (these simulations use $n=10^{5}$ grains). The variation of $\beta$ and $\beta^{\prime}$ with the aspect ratio $\lambda$ (minor axis length/major axis length) is shown in Fig. 4. For the RAP case, we observe an initial flat region, where the ellipses have a $\beta$ value close to that of circles. For larger ellipticities, we see a very rapid drop off in $\beta$, with $\beta=0.124$ (2) found for $\lambda=0.1$. Simulations using the RRAP model again show a very different behavior for $\beta^{\prime}$. There is an initial rapid increase in $\beta^{\prime}$ with ellipticity, with a maximum value of $\beta^{\prime}=0.359$ (3) found for $\lambda=0.5$. Above this value there is a slow drop off in $\beta^{\prime}$, with $\beta^{\prime}=0.341(2)$ found for $\lambda=0.1$.

The packing efficiency exponents $\beta$ and $\beta^{\prime}$ are measures of how quickly the various shapes are able to fill in space in the RAP and RRAP models. Larger packing efficiency exponents are associated with packings that on average have grains with a larger area at a given step in the packing process. To determine the relationship between the packing efficiency and the shape of the grain we need to consider how the area of the grain is constrained in each packing model. We can achieve this by identifying where contacts between grains typically occur. This provides us with a constraining length $D_{c}$ associated with the contact points. In the RAP model, the growth of the grains is constrained by a single contact between the growing grain and an already placed grain. For straight edged convex grains, collisions in the RAP model in general occur between a vertex (which is at the farthest distance $D_{c}=D_{\max }$ from

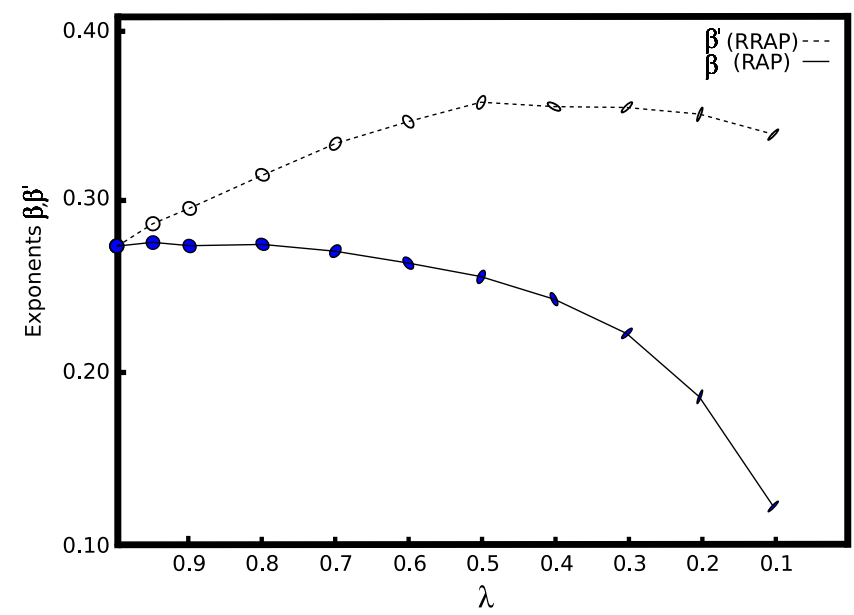

FIG. 4 (color online). Variation of packing efficiency exponents $\beta$ (filled symbols) and $\beta^{\prime}$ (open symbols) for RAP and RRAP packings of ellipses with ellipticities from $\lambda=1$ (circle) to $\lambda=0.1$ (highly elliptical). the center of the grain) and the edge of the other grain. (The only other possibilities would be a collision between two grains exactly at their respective vertices or with both grains aligned edge to edge, both of which would require the orientation of the grains to be identical and so have infinitesimal probability to occur.) Contrarily, ellipses in the RAP model will contact at $D_{\max }$ only when one grain is oriented at exactly matching angles to the surface of the other grain. In general, collisions between ellipses will occur at arbitrary points on each ellipses surface. Thus in this case the constraining length $D_{c}$ is the average distance from the center of the ellipse to its boundary $D_{c}=D_{\mathrm{av}}=$ $\frac{2 a}{\pi} K\left(1-\lambda^{-2}\right)$. (Here $K$ is an elliptic integral of the second kind and $a$ is the length of the minor axis.)

The situation is very different for the RRAP model. A grain of any shape is able to continue growing and rotating until the point that is a minimum distance $\left(D_{c}=D_{\min }\right)$ from the center of the grain is constrained by the pore wall. The other possibility is that the grain cannot achieve this maximal area, because it gets constrained by contacting with two separate grains. The likelihood of this second case will be much higher for grains with a larger anisotropy (ratio between $D_{\min }$ and $D_{\max }$ ). This has been verified to be the case: we measured 38\% of grains in RRAP packings of triangles failing to achieve their maximum size compared to only $18 \%$ for squares and $16 \%$ for octagons.

Figure 5 shows the relative sizes of several polygonal grain shapes with the same $D_{\max }$ and $D_{\min }$ lengths. Grains contacting at $D_{c}=D_{\max }$ have the smallest area and conversely grains contacting at $D_{c}=D_{\min }$ have the largest area and this leads to smaller packing efficiency exponents in the RAP case with respect to the RRAP case. But what about the shape dependence? We can see that the area decreases with the number of sides when $D_{\min }$ is constrained and instead it increases when $D_{\max }$ is constrained. If the grain area at fixed $D_{c}$ is the parameter controlling packing efficiency, this should be quantitatively reflected in the values of $\beta$ and $\beta^{\prime}$. This is indeed confirmed by plotting the packing efficiency exponents $\beta$ and $\beta^{\prime}$ against the area of the grain using $D_{c}=D_{\max }=1$ for the RAP model and $D_{c}=D_{\min }=1$ for the RRAP model (see Fig. 6). We observe that all our data for $\beta$ and $\beta^{\prime}$ for all

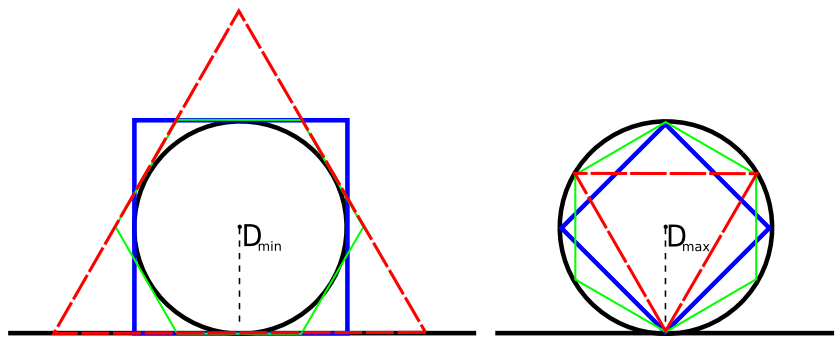

FIG. 5 (color online). Relative sizes of a triangle, square, hexagon, and circle when each grain's minimum distance $D_{\text {min }}$ (left) and maximum distance $D_{\max }$ (right) from its center to its edge is the same. 


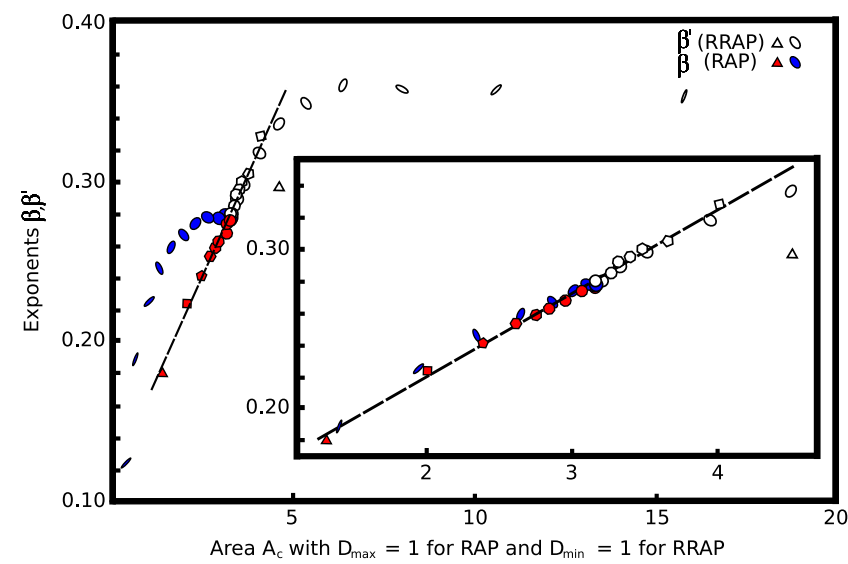

FIG. 6 (color online). Packing efficiency exponents $\beta$ (filled symbols) and $\beta^{\prime}$ (open symbols) versus the area of the grain for the RAP model with $D_{\max }=1$ and for the RRAP model with $D_{\min }=1$. Inset shows data rescaled for the ellipses in the RAP model, using $D_{\mathrm{av}}=1$, following the same universal trend. Dashed line is a best fit to the data (slope $m=0.05, y$ intercept $c=0.12$ ).

the polygonal shapes follow a universal trend, with all the data in the RAP model lying on an almost straight line which is continued for the data for the RRAP model. Also, as expected, the triangular shape (which has the largest anisotropy) in the RRAP model is below the trend, as its packing efficiency is reduced due to the high percentage of grains that are unable to achieve their maximum area. By appropriately rescaling the RAP data for ellipses with $D_{c}=D_{\mathrm{av}}=1$ (inset of Fig. 6), we see that they also collapse onto the same line as the data for the straight edged grains and this trend is continued for ellipses with low anisotropy $(\lambda>0.7)$ in the RRAP model. Such a universal linear relation which holds for a large variety of grain shapes, grain anisotropies, and for two different models is a remarkable discovery which allows us to quantitatively predict the scaling exponents. Specifically, we obtain $\beta \simeq 0.05 A_{c}+0.12$. Here $A_{c}$ is the grain's area with $D_{c}=D_{\max }=1$ for polygons in the RAP model, with $D_{c}=D_{\min }=1$ for polygons and ellipses in the RRAP model, and $D_{c}=D_{\mathrm{av}}=1$ for ellipses in the RAP model.

Packing models of the kind considered here have an applicability to a wide variety of biological and physical problems [12-14] and also find applications in the study of advanced materials $[5,15]$. Our inclusion of the important effect of grain rotations opens an interesting avenue to investigate the role shape plays in determining the properties of such packings.

In conclusion, we have demonstrated the strong dependence of the scaling properties on the grain shape in the RAP and RRAP models. The observed trends in the packing efficiency are complex and nonlinear. We have, however, discovered that these complex behaviors are well described by a simple linear relation (with the exception of shapes with very large anisotropies). Of all the shapes we have considered, the highest packing efficiency $\beta^{\prime}=$ 0.359 (3) was found for ellipses with $\lambda \sim 0.5$ in the RRAP model. This may be related to the large packing fractions (above those observed for circles) that have been found in 2D random packings of ellipses [16]. Similar results have also been observed for packings of ellipsoids [17]. From the shapes we considered, the circle was found to have the largest packing efficiency in the RAP model and conversely the lowest in the RRAP model. It remains an open question as to whether the circle is indeed the most efficient shape in the RAP model; however, clearly shapes with lower packing efficiencies exist in the RRAP model, as one need only consider the reduction in the width of a grain and the resulting tendency toward a line with zero area and zero packing efficiency. In future work we will examine other grain shapes, including irregular nonconvex grains and extend our models to three dimensions.

This work was partially supported by the ARC project DP0450292 and the Australian Partnership for Advanced Computing National Facilities. We acknowledge support of the SFI/HEA Irish Centre for High-End Computing and the Trinity Center for High Performance Computing.

[1] B. B. Mandelbrot, The Fractal Geometry of Nature (Oxford, New York, 1983).

[2] N. V. Brilliantov, Y. A. Andrienko, P. L. Krapivsky, and J. Kurths, Phys. Rev. Lett. 76, 4058 (1996).

[3] E. Ben-Naim and P. L. Krapivsky, Phys. Rev. E 54, 3562 (1996).

[4] M. Wackenhut and H. Herrmann, Phys. Rev. E 68, 041303 (2003).

[5] T. Aste and D. Weaire, The Pursuit of Perfect Packing (Taylor \& Francis, Boca Raton, FL, 2008), 2nd ed.

[6] S. S. Manna and H. J. Herrmann, J. Phys. A 24, L481 (1991).

[7] S. S. Manna, Physica (Amsterdam) 187A, 373 (1992).

[8] N. V. Brilliantov, P.L. Krapivsky, and Y. A. Andrienko, J. Phys. A 27, L381 (1994).

[9] Y. A. Andrienko, N. V. Brilliantov, and P. L. Krapivsky, J. Stat. Phys. 75, 507 (1994).

[10] Y.A. Andrienko, N. V. Brilliantov, and J. Kurths, Eur. Phys. J. B 15, 539 (2000).

[11] P.S. Dodds and J.S. Weitz, Phys. Rev. E 65, 056108 (2002).

[12] P.S. Dodds and J.S. Weitz, Phys. Rev. E 67, 016117 (2003).

[13] T. Aste, Phys. Rev. E 53, 2571 (1996).

[14] R. Mahmoodi Baram and H. J. Herrmann, Phys. Rev. Lett. 95, 224303 (2005).

[15] J. S. Andrade, H. J. Herrmann, R. F. S. Andrade, and L. R. da Silva, Phys. Rev. Lett. 94, 018702 (2005).

[16] G. Delaney, D. Weaire, S. Hutzler, and S. Murphy, Philos. Mag. Lett. 85, 89 (2005).

[17] A. Donev, I. Cisse, D. Sachs, E. Variano, F. H. Stillinger, R. Connelly, S. Torquato, and P. M. Chaikin, Science 303, 990 (2004). 